\title{
Estadística de egresos hospitalarios del sector público del Sistema Nacional de Salud, 2003
}

C omo ha sido tradición desde hace hace más de una década, cada año la Dirección General en Información en Salud ofrece a todos los investigadores e interesados en la morbilidad y mortalidad hospitalaria la información que genera el Sistema Nacional de Salud.

Esta información comprende los egresos hospitalarios registrados en personas que ocuparon una cama censable en los hospitales generales y especializados de las instituciones públicas del sector salud: Secretaría de Salud [SSA] (incluye Institutos Nacionales de Salud y el Hospital General de México), Instituto Mexicano del Seguro Social (IMSS), tanto régimen ordinario como régimen de seguridad social, Instituto de Seguridad y Servicios Sociales de los Trabajadores del Estado (ISSSTE), Petróleos Mexicanos, Secretaría de la Defensa Nacional (SEDENA) y Secretaría de Marina.

Al igual que en 2002, se utiliza la clasificación que toma como base la Lista Mexicana II para morbilidad y mortalidad y que permite la agrupación de causas en tres grandes rubros: a) enfermedades transmisibles, maternas, perinatales y nutricionales, b) enfermedades no transmisibles y c) lesiones, considerando dos apartados: otras causas de contacto con los servicios de salud, y causas mal definidas.

En el año 2003 se registraron 4353429 egresos, 135520 más que el año 2002, lo que representa 3.2\% de incremento; $69 \%$ de los egresos atendidos fueron mujeres y $31 \%$ correspondió a los hombres. Si revisamos la distribución por agrupación de instituciones que atendieron población no derechohabiente y derechohabiente, se registraron $44 \%$ y $56 \%$ de los egresos, respectivamente; en ambos grupos se mantiene la relación de mayor porcentaje de egresos del sexo femenino.

El grupo relacionado con las enfermedades transmisibles, maternas, perinatales y nutricionales representa $44.5 \%$ del total de egresos, en segundo lugar se encuentran las enfermedades no transmisibles con $43 \%$ y lesiones con $7.3 \%$. Sin embargo, si eliminamos los egresos por causas maternas, el primer grupo representa sólo $11.1 \%$ del total de los egresos hospitalarios.

Del total de los egresos 114255 fueron por defunción (52\% hombres y $48 \%$ mujeres). El Grupo II de enfermedades no transmisibles es el que concentra el mayor porcentaje de mortalidad hospitalaria tanto para hombres (35.8\%) como para mujeres $(35.6 \%)$, le sigue en orden de importancia el Grupo I de enfermedades transmisibles, maternas, perinatales y nutricionales (11.5\% y $9.1 \%$ ) y el grupo de lesiones $(2.6 \%$ y $1.1 \%$, respectivamente) ocupa el último lugar.

Existen patrones distintos cuando analizamos la mortalidad hospitalaria por causas, grupos de edad y sexo como se aprecian en los cuadros XV y XVI. Como ejemplo, en enfermedades no transmisibles, el grupo de edad de 45 a 59 años en los hombres, concentra $20.2 \%$ de muertes; en las mujeres el mayor porcentaje por esta causa (41\%), está en el grupo de 60 años y más.

Los 4.3 millones de egresos generaron un total de 17868734 de días estancia, de ellos, 10 millones aproximadamente se dieron por la atención a mujeres, y correspondieron 2861 4763 a la atención de causas maternas. Los mayores números de días estancia se dan en las instituciones que atienden población derechohabiente donde el IMSS registra 78\% de ellos; por su parte, en las instituciones que atienden población no derechohabiente la SSA cubre $92 \%$ de los días estancia. En todas las instituciones el porcentaje de días estancia es mayor para el grupo de las mujeres.

El IMSS, con la mayor capacidad instalada de las instituciones que atienden población derechohabiente, contó con $80.2 \%$ de los egresos hospitalarios, a su vez dicha institución reportó 8479830 días estancia (78\%); por su parte, la SSA contó con 1707909 (89\%) de los egresos de la población no derechohabiente con 6469385 (92\%) de días estancia.

El promedio de días estancia es utilizado como un indicador de eficiencia, el valor nacional es de 4.1 días, con una diferencia de un día de 
estadía entre la población derechohabiente y no derechohabiente. Las instituciones con mayor promedio de días estancia son ISSSTE 5.3, IMSS 4.4, Sedena 4.2 y SSA 3.8. La diferencia entre el ISSSTE y la de menor pro- medio IMSS-Oportunidades (2.7), es de 2.6 días estancia.

Este anexo incluye ocho cuadros para morbilidad y ocho cuadros de mortalidad hospitalaria, con desglose por sexo, población derecho- habiente y no derechohabiente, por institución y grupos de edad. Para mayor información consultar el Boletín de Información Estadística, Volumen II, Daños a la Salud, en el sitio http://sinais.salud.gob.mx. 


\section{Cuadro I \\ Grandes grupos de causas de egresos hospitalarios, población derechohabiente.* Hombres. México, 2003}

Causas

nutricionales

Egresos Tasa por $100000^{\ddagger} \quad \%$

Total

$861004 \quad 3695 \quad 100.0$

I. Enfermedades transmisibles, maternas, perinatales y nutricionales

\begin{tabular}{rrr}
141462 & 607 & 16.4 \\
\hline 38822 & 167 & 4.5 \\
\hline 44523 & 191 & 5.2 \\
\hline 53137 & 228 & 6.2 \\
\hline 4980 & 21 & 0.6
\end{tabular}

II. Enfermedades no transmisibles

\begin{tabular}{l}
\hline Tumores malignos \\
\hline O tros tumores \\
\hline Diabetes mellitus \\
\hline Enfermedades endocrinas, metabólicas, hematológicas e inmunológicas (excepto diabetes mellitus)
\end{tabular}

Trastornos mentales y enfermedades del sistema nervioso
Enfermedades de los órganos de los sentidos

Enfermedades cardiovasculares
Enfermedades respiratorias

Enfermedades digestivas 123

Enfermedades del sistema génito urinario
Enfermedades de la piel

Enfermedades del sistema músculo esquelético

Anomalías congénitas

Enfermedades de la boca

\begin{tabular}{rrr}
545495 & 2341 & 63.4 \\
\hline 39735 & 171 & 4.6 \\
\hline 12095 & 52 & 1.4 \\
\hline 37373 & 160 & 4.3 \\
\hline 9961 & 43 & 1.2 \\
\hline 20968 & 90 & 2.4 \\
\hline 13796 & 59 & 1.6 \\
\hline 80710 & 346 & 9.4 \\
\hline 44696 & 192 & 5.2 \\
\hline 123557 & 530 & 14.4 \\
\hline 89139 & 383 & 10.4 \\
\hline 14561 & 62 & 1.7 \\
\hline 38754 & 166 & 4.5 \\
\hline 18035 & 77 & 2.1 \\
\hline 2115 & 9 & 0.2
\end{tabular}

III. Causas externas de morbilidad y mortalidad

Accidentes

Lesiones intencionales

Eventos (lesiones) de intención no determinada

Fracturas

Amputaciones

103776

Luxaciones

Traumatismos

Heridas

Q uemaduras

Envenenamiento

Traumatismos, complicaciones

Demás traumatismos

\begin{tabular}{rrr}
103776 & 445 & 12.1 \\
\hline 0 & 0 & 0.0 \\
0 & 0 & 0.0 \\
0 & 0 & 0.0 \\
\hline 50779 & 218 & 5.9 \\
\hline 1984 & 9 & 0.2 \\
\hline 5382 & 23 & 0.6 \\
\hline 22899 & 98 & 2.7 \\
\hline 6836 & 29 & 0.8 \\
\hline 3874 & 17 & 0.4 \\
\hline 1727 & 7 & 0.2 \\
\hline 8973 & 39 & 1.0 \\
\hline 1322 & 6 & 0.2 \\
& & \\
23057 & 99 & 2.7 \\
\hline 35041 & 150 & 4.1 \\
\hline 12173 & 52 & 1.4
\end{tabular}

Causas mal definidas

0 tras causas de contacto con los servicios de salud

No especificado

* Incluye: Instituto Mexicano del Seguro Social, Instituto de Seguridad y Servicios Sociales de los Trabajadores del Estado y Secretaría de la Defensa $\mathrm{N}$ acional

* Estimación realizada a partir de la muestra censal del Censo 2000 y de las proyecciones de la población de México 2000-2030. Consejo N acional de Población, 2003

Fuente: Secretaría de Salud. D irección General de Información en Salud. Base de datos de egresos hospitalarios. México, 2003 


\section{Cuadro II \\ Grandes grupos de causas de egresos hospitalarios, población derechohabiente.* Mujeres. México, 2003}

Causas

Egresos

Total

$\begin{array}{lll}1571927 & 6563 & 100\end{array}$

I. Enfermedades transmisibles, maternas, perinatales y nutricionales Enfermedades infecciosas y parasitarias Infecciones respiratorias

$701020 \quad 2927 \quad 44.6$

Causas maternas

$\begin{array}{lll}44781 & 187 & 2.8\end{array}$

Ciertas afecciones originadas en el periodo perinatal

37393

$156 \quad 2.4$

D eficiencias de la nutrición

568537

2374

43142

180

7167

30

Enfermedades no transmisibles

$708262 \quad 2957 \quad 45.1$

Tumores malignos

0 tros tumores

\begin{tabular}{rrr}
708262 & 2957 & 45.1 \\
\hline 48967 & 204 & 3.1 \\
\hline 61226 & 256 & 3.9 \\
\hline 47062 & 196 & 3.0 \\
\hline 15112 & 63 & 1.0 \\
\hline 23390 & 98 & 1.5 \\
\hline 15621 & 65 & 1.0 \\
\hline 82942 & 346 & 5.3 \\
\hline 41821 & 175 & 2.7 \\
\hline 163012 & 681 & 10.4 \\
\hline 131066 & 547 & 8.3 \\
\hline 13854 & 58 & 0.9 \\
\hline 45435 & 190 & 2.9 \\
\hline 16332 & 68 & 1.0 \\
\hline 2422 & 10 & 0.2
\end{tabular}

III. Causas externas de morbilidad y mortalidad

$66971 \quad 280 \quad 4.3$

\begin{tabular}{lrrr}
\hline Accidentes & 0 & 0 & 0.0 \\
\hline Lesiones intencionales & 0 & 0 & 0.0 \\
\hline Eventos (lesiones) de intención no determinada & 0 & 0 & 0.0 \\
\hline Fracturas & 34706 & 145 & 2.2 \\
\hline Amputaciones & 752 & 3 & 0.0 \\
\hline Luxaciones & 2950 & 12 & 0.2 \\
\hline Traumatismos & 10248 & 43 & 0.7 \\
\hline Heridas & 2389 & 10 & 0.2 \\
\hline Q uemaduras & 1899 & 8 & 0.1 \\
\hline Envenenamiento & 1643 & 7 & 0.1 \\
\hline Traumatismos, complicaciones & 11296 & 47 & 0.7 \\
\hline Demás traumatismos & 1088 & 5 & 0.1 \\
& & 24219 & 101 \\
Causas mal definidas & 54225 & 226 & 1.5 \\
\hline tras causas de contacto con los servicios de salud & 17230 & 72 & 1.1
\end{tabular}

* Incluye: Instituto Mexicano del Seguro Social, Instituto de Seguridad y Servicios Sociales de los Trabajadores del Estado y Secretaría de la Defensa Nacional

₹ Estimación realizada a partir de la muestra censal del Censo 2000 y las proyecciones de la población de México 2000-2030. Consejo Nacional de Población, 2003

Fuente: Secretaría de Salud. Dirección General de Información en Salud. Base de datos de egresos hospitalarios. México, 2003 


\section{Cuadro III \\ Grandes grupos de causas de egresos hospitalarios, población no derechohabiente.* Hombres. MÉxico, 2003}

Causas

nutricionales

Egresos Tasa por $100000^{\ddagger} \quad \%$

Total

$498304 \quad 1746 \quad 100$

I. Enfermedades transmisibles, maternas, perinatales y nutricionales

\begin{tabular}{rrr}
112366 & 394 & 22.5 \\
\hline 29999 & 105 & 6.0 \\
\hline 29757 & 104 & 6.0 \\
\hline 48982 & 172 & 9.8 \\
\hline 3628 & 13 & 0.7
\end{tabular}

II. Enfermedades no transmisibles

\begin{tabular}{rrr}
269955 & 946 & 54.2 \\
\hline 15503 & 54 & 3.1 \\
\hline 6823 & 24 & 1.4 \\
\hline 17218 & 60 & 3.5 \\
\hline 4831 & 17 & 1.0 \\
\hline 23320 & 82 & 4.7 \\
\hline 7150 & 25 & 1.4 \\
\hline 26269 & 92 & 5.3 \\
\hline 20499 & 72 & 4.1 \\
\hline 76351 & 268 & 15.3 \\
\hline 37693 & 132 & 7.6 \\
\hline 9145 & 32 & 1.8 \\
\hline 8908 & 31 & 1.8 \\
\hline 14614 & 51 & 2.9 \\
\hline 1631 & 6 & 0.3
\end{tabular}

III. Causas externas de morbilidad y mortalidad

\begin{tabular}{rrr}
97789 & 343 & 19.6 \\
\hline 37689 & 132 & 7.6 \\
\hline 1219 & 4 & 0.2 \\
\hline 3086 & 11 & 0.6 \\
\hline 28453 & 100 & 5.7 \\
\hline 13205 & 46 & 2.6 \\
\hline 4400 & 15 & 0.9 \\
\hline 5952 & 21 & 1.2 \\
\hline 2453 & 9 & 0.5 \\
1332 & 5 & 0.3
\end{tabular}

0 tras causas de contacto con los servicios de salud

\begin{tabular}{rrr}
9949 & 34.86 & 2.0 \\
\hline 7734 & 27.10 & 1.6 \\
\hline 511 & 1.79 & 0.1
\end{tabular}

No especificado

511

* Incluye: Secretaría de Salud e IMSS O portunidades

₹ Estimación realizada a partir de la muestra censal del Censo 2000 y las proyecciones de la población de México 2000-2030. Consejo Nacional de Población, 2003

Fuente: Secretaría de Salud. Dirección General de Información en Salud. Base de datos de egresos hospitalarios. México, 2003 


\section{Cuadro IV \\ Grandes Grupos de CAUSAS de egresos hospitalarios, población no derechohabiente.* Mujeres. México, 2003}

Causas

Egresos Tasa por $100000^{\ddagger} \%$

Total

$\begin{array}{lll}1421931 & 5004 & 100.0\end{array}$

I. Enfermedades transmisibles, maternas, perinatales y nutricionales

$985373 \quad 3468 \quad 69.3$

Enfermedades infecciosas y parasitarias

$\begin{array}{rrr}30999 & 109 & 2.2 \\ 24313 & 86 & 1.7\end{array}$

Infecciones respiratorias

Causas maternas

$\begin{array}{lll}885819 & 3117 & 62.3\end{array}$

Ciertas afecciones originadas en el periodo perinatal

39373

139

Deficiencias de la nutrición

$\begin{array}{lll}4869 & 17 & 0.3\end{array}$

II. Enfermedades no transmisibles

$\begin{array}{lll}345476 & 1216 & 24.3\end{array}$

Tumores malignos

$\begin{array}{lll}23511 & 83 & 1.7\end{array}$

O tros tumores
Diabetes mellitus

Enfermedades endocrinas, metabólicas, hematológicas e inmunológicas

$33110 \quad 117 \quad 2.3$

Trastornos mentales y enfermedades del sistema nervioso

$\begin{array}{lll}24022 & 85 & 1.7\end{array}$

Enfermedades de los órganos de los sentidos

$\begin{array}{lll}7472 & 26 & 0.5\end{array}$

Enfermedades cardiovasculares

$15302 \quad 54 \quad 1.1$

Enfermedades respiratorias

Enfermedades digestivas

$\begin{array}{lll}8648 & 30 & 0.6\end{array}$

Enfermedades del sistema génito urinario

29252

20300

$103 \quad 2.1$

Enfermedades de la pie

Enfermedades del sistema músculo esquelético

93151

57334

862

11113

Anomalías congénitas

11914

Enfermedades de la boca

1726

71

$328-66$

III. Causas externas de morbilidad y mortalidad

\begin{tabular}{rrr}
49009 & 172 & 3.4 \\
19477 & 69 & 1.4 \\
361 & 1 & 0.0 \\
1639 & 6 & 0.1 \\
\hline 11609 & 41 & 0.8 \\
\hline 3575 & 13 & 0.3 \\
\hline 2601 & 9 & 0.2 \\
\hline 5237 & 18 & 0.4 \\
\hline 3537 & 12 & 0.2 \\
973 & 3 & 0.1
\end{tabular}

0 tras causas de contacto con los servicios de salud

\begin{tabular}{rrr}
32801 & 115 & 2.3 \\
\hline 7978 & 28 & 0.6 \\
\hline 1294 & 5 & 0.1
\end{tabular}

No especificado

1294

$202 \quad 4.0$

Fracturas

Amputaciones

Luxaciones

Traumatismos

Q uemaduras

Envenenamiento

Traumatismos, complicaciones

Demás traumatismos

* Incluye: Secretaría de Salud e IMSS O portunidades

* Estimación realizada a partir de la muestra censal del Censo 2000 y las proyecciones de la población de México 2000-2030. Consejo Nacional de Población, 2003

Fuente: Secretaría de Salud. Dirección General de Información en Salud. Base de datos de egresos hospitalarios. México, 2003 


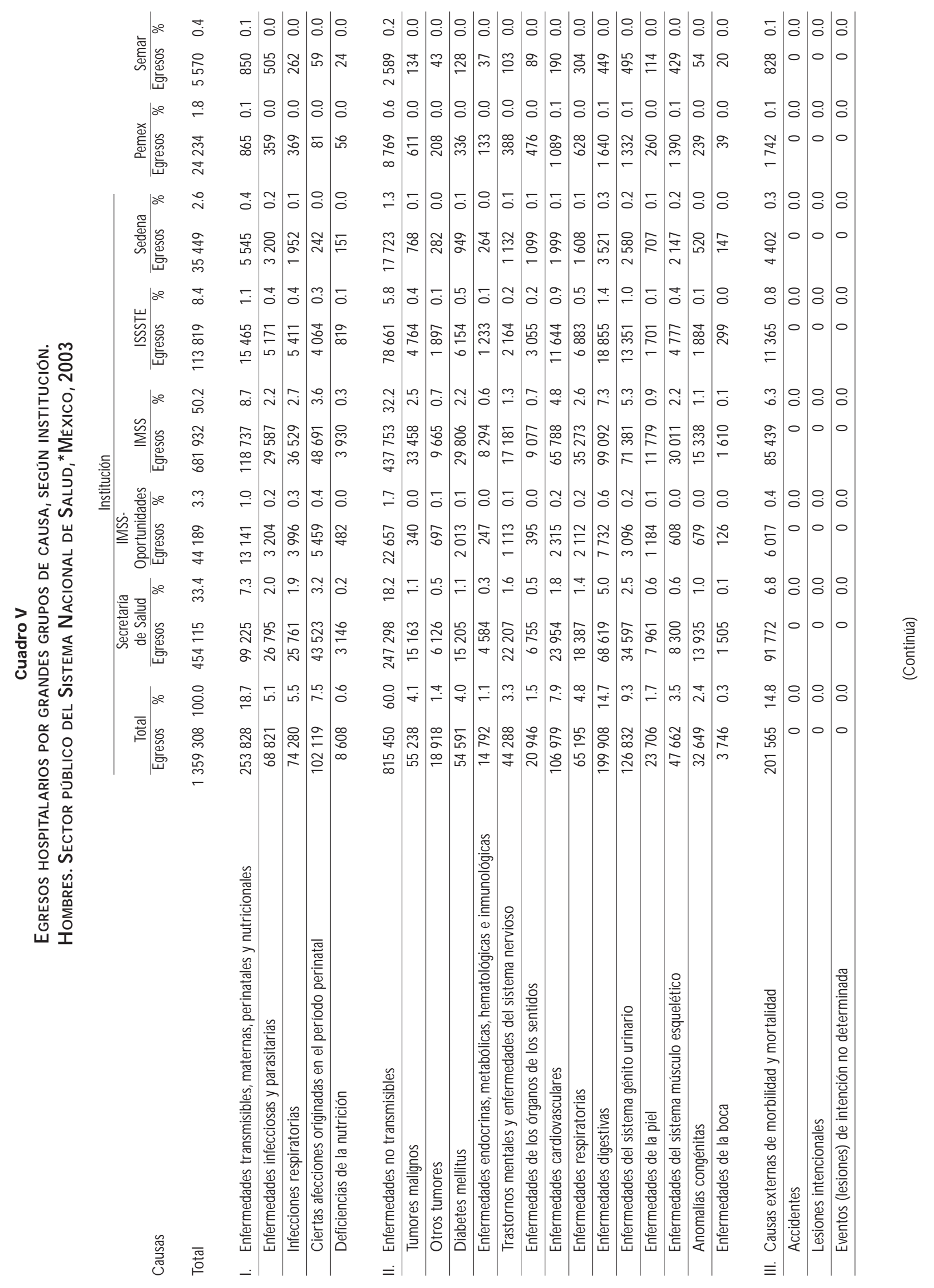




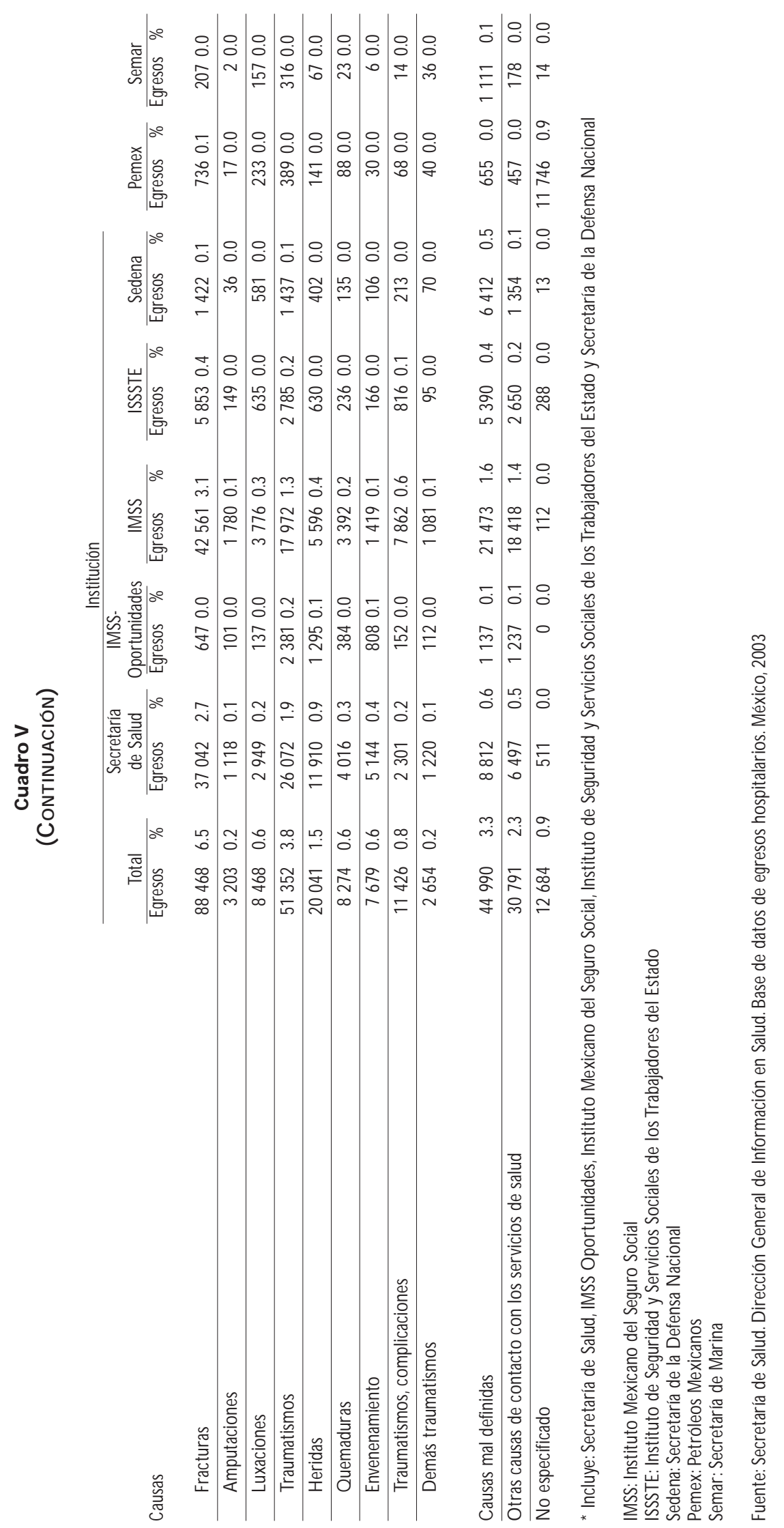




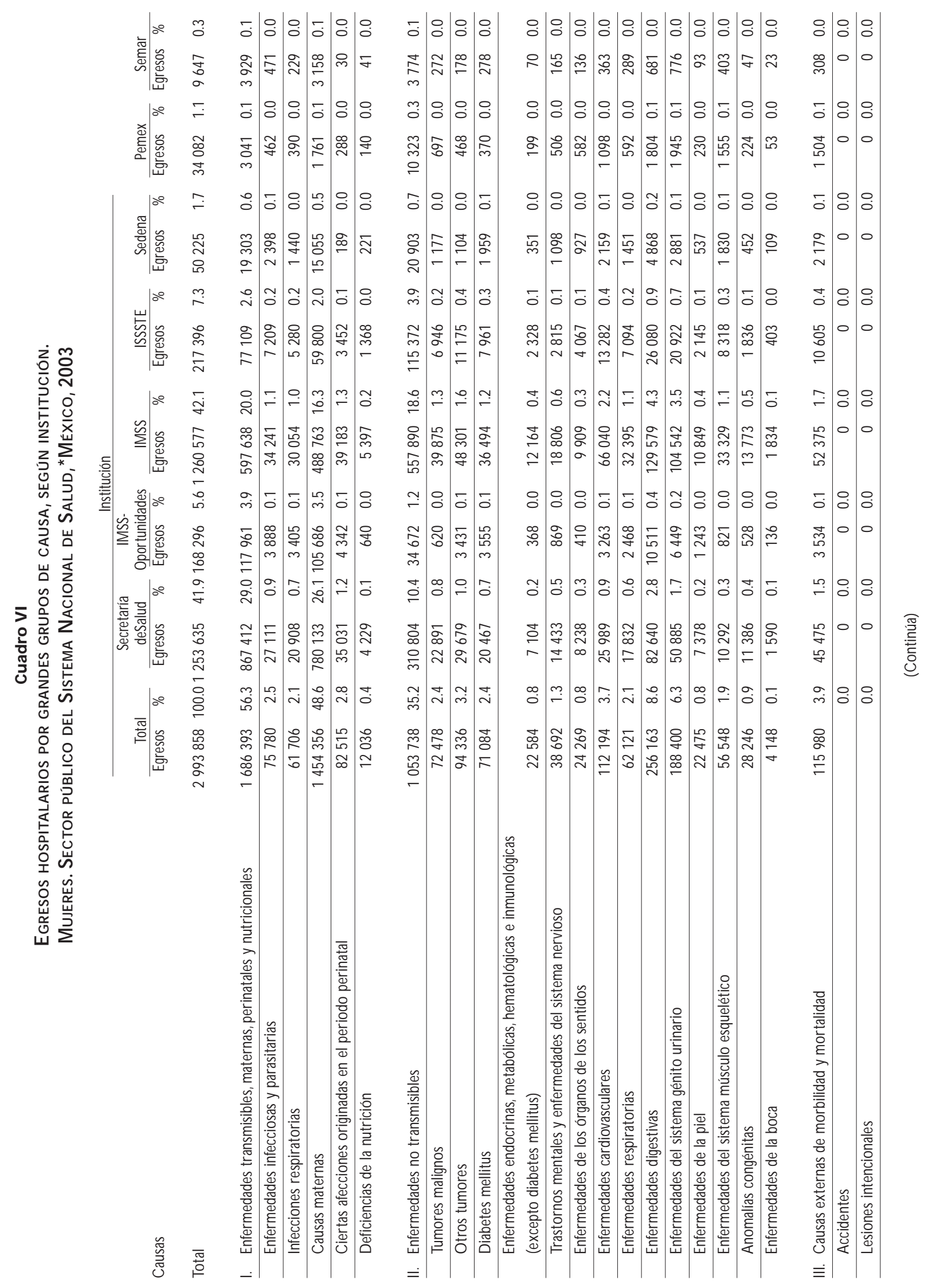




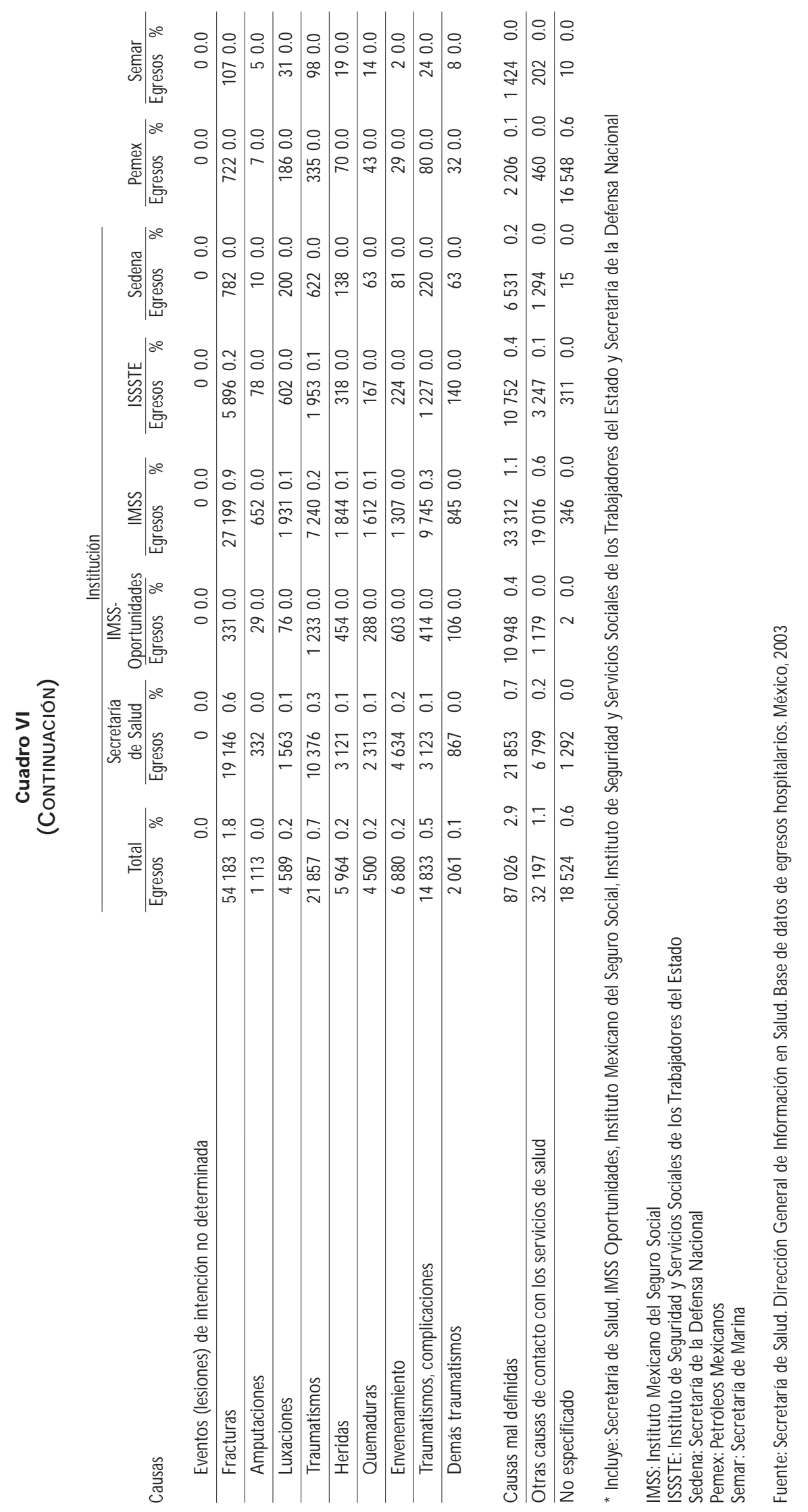




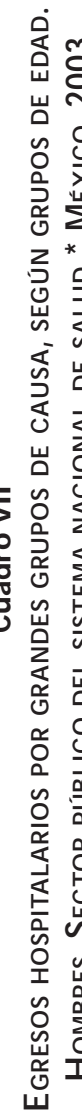

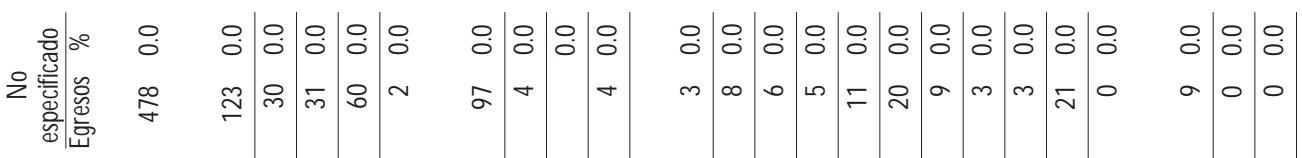

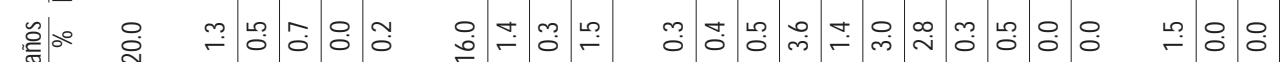

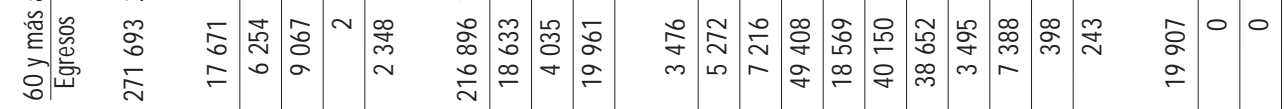

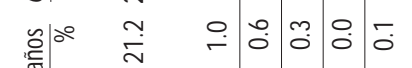

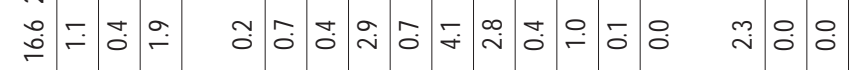

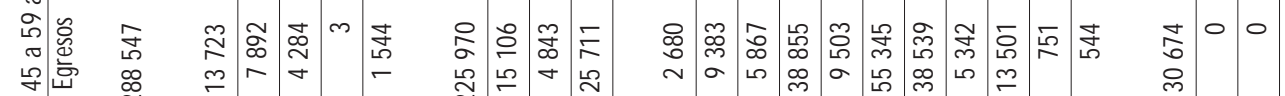

है।

高

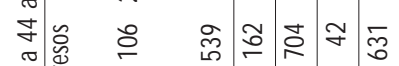

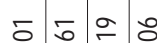

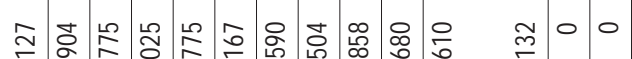

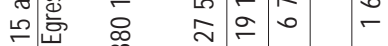

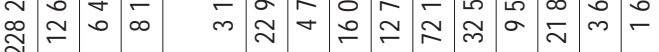

㽝

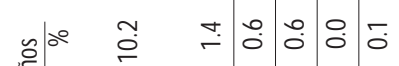

กิ่

ஸ̃

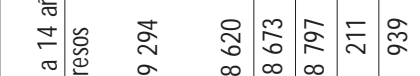

角

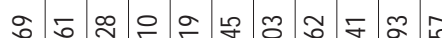

$\stackrel{9}{m} 00$

$\therefore$ in $N$

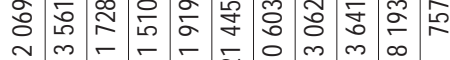

峞

$8 \mid$

i)

$\begin{array}{llll}4 & 2 & -4 & 0\end{array}$

न

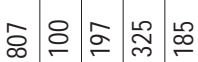

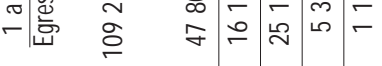

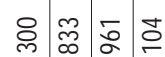

두용

\begin{tabular}{l|l|l}
$\stackrel{\infty}{m}$ & 0 & 0 \\
$N$ & &
\end{tabular}

$\stackrel{m}{y} \stackrel{\infty}{\sim}$

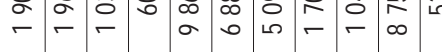

वे.

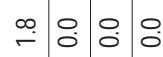

ન્વ

ำ

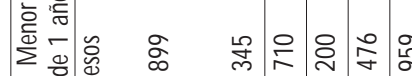

워

品

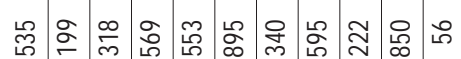

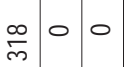

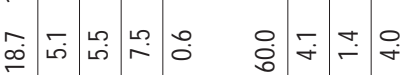

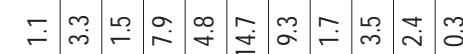

文

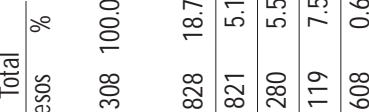

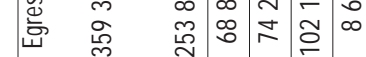

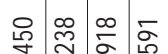

ริ

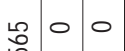

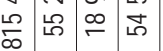

ป 寸

กั
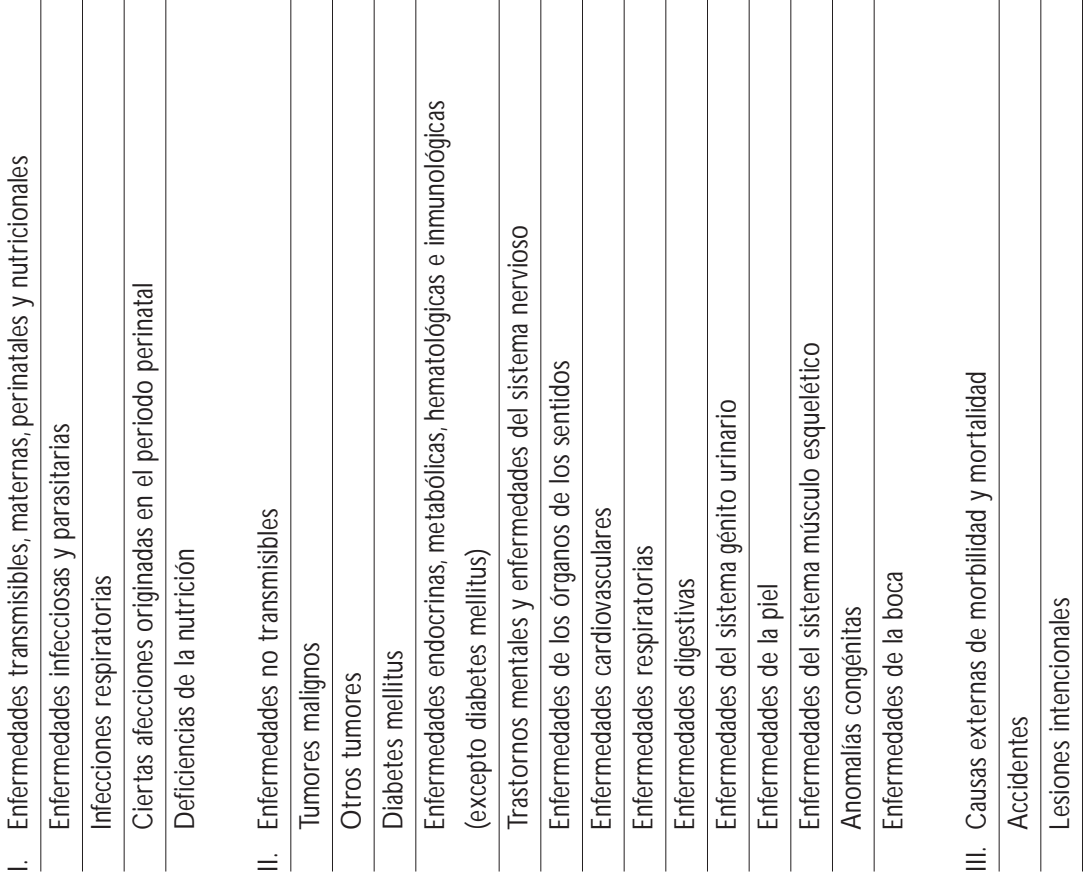


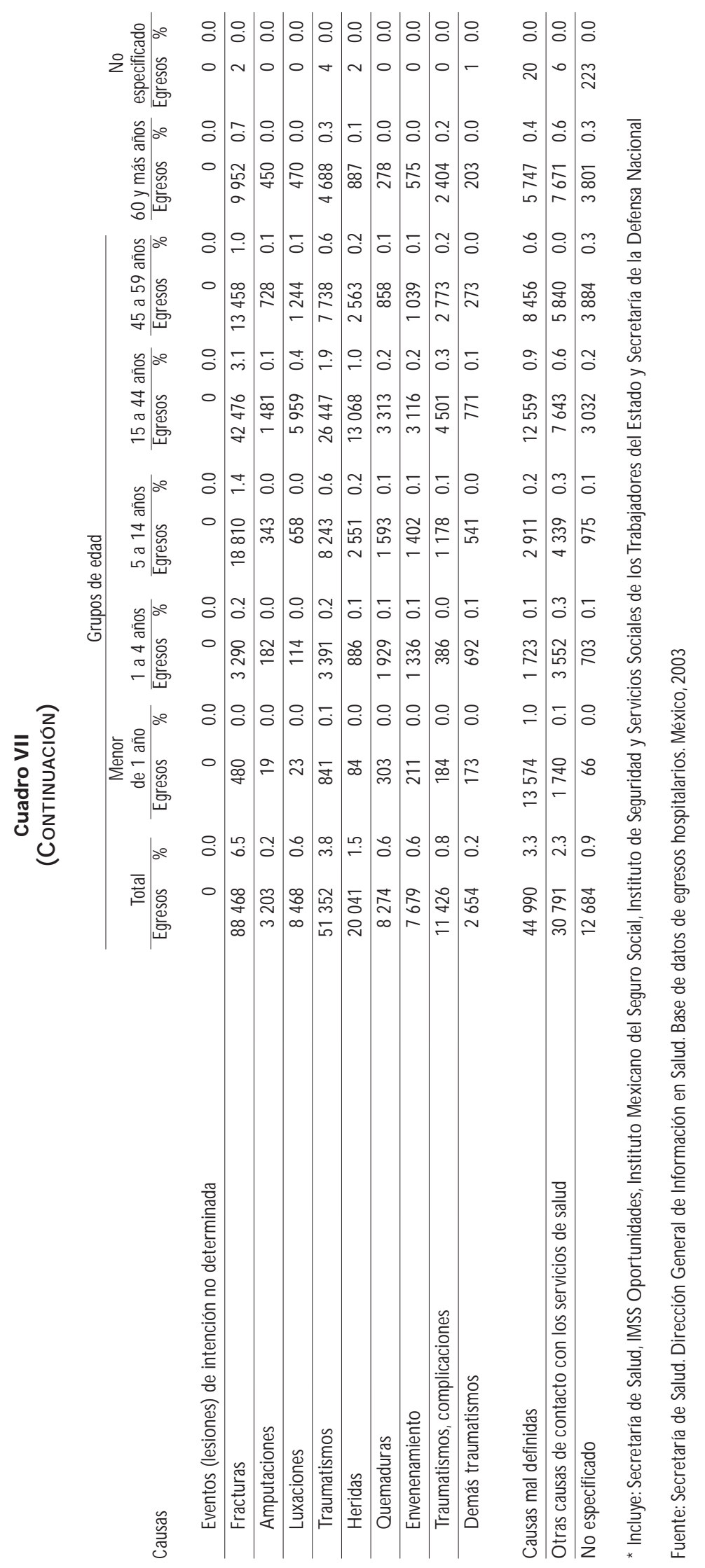




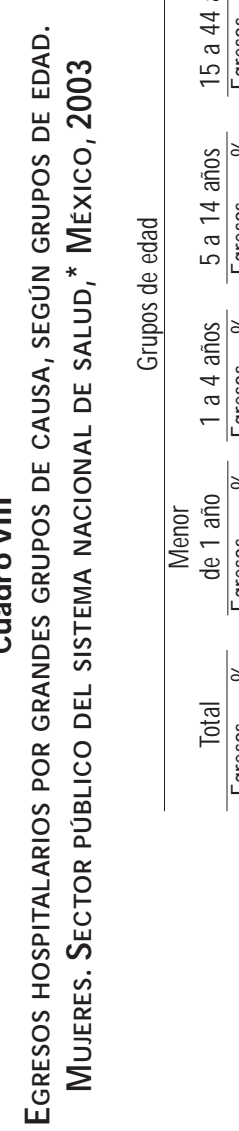

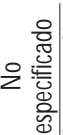

案

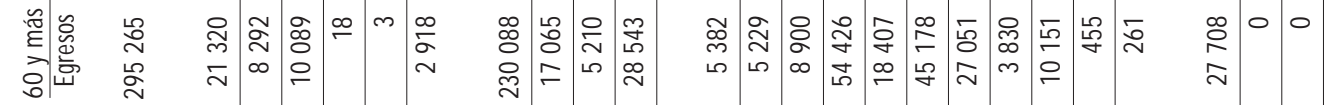

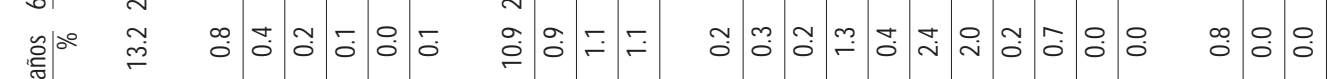

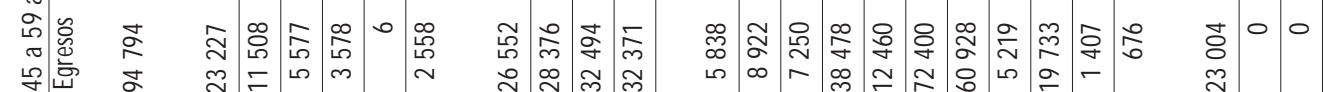

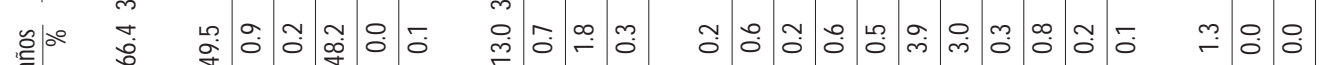

西多

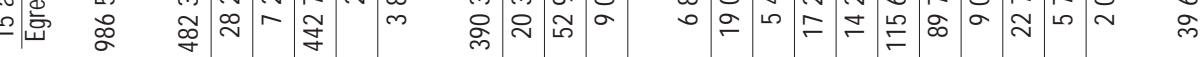

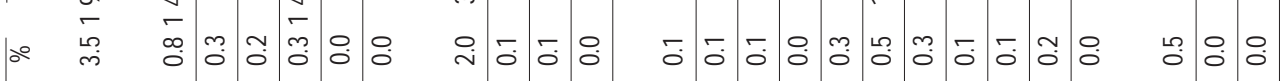

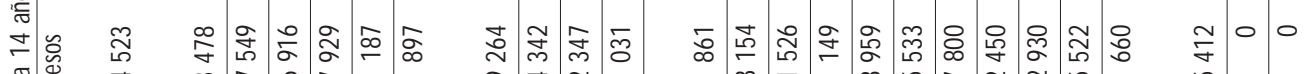
ம

:

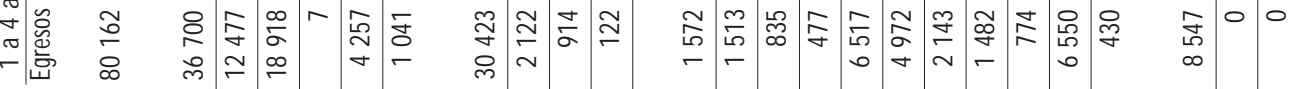

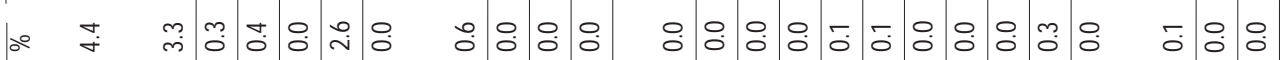

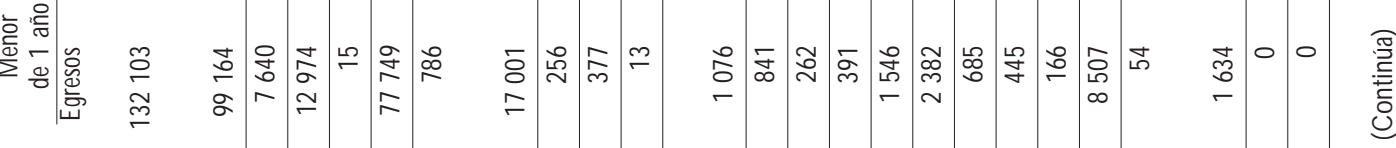

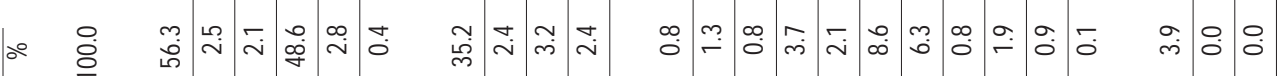

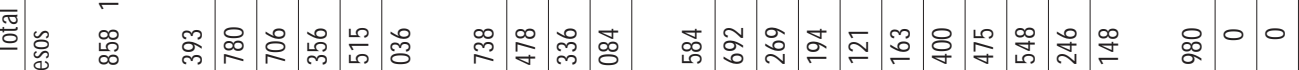

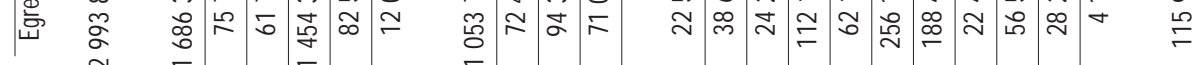
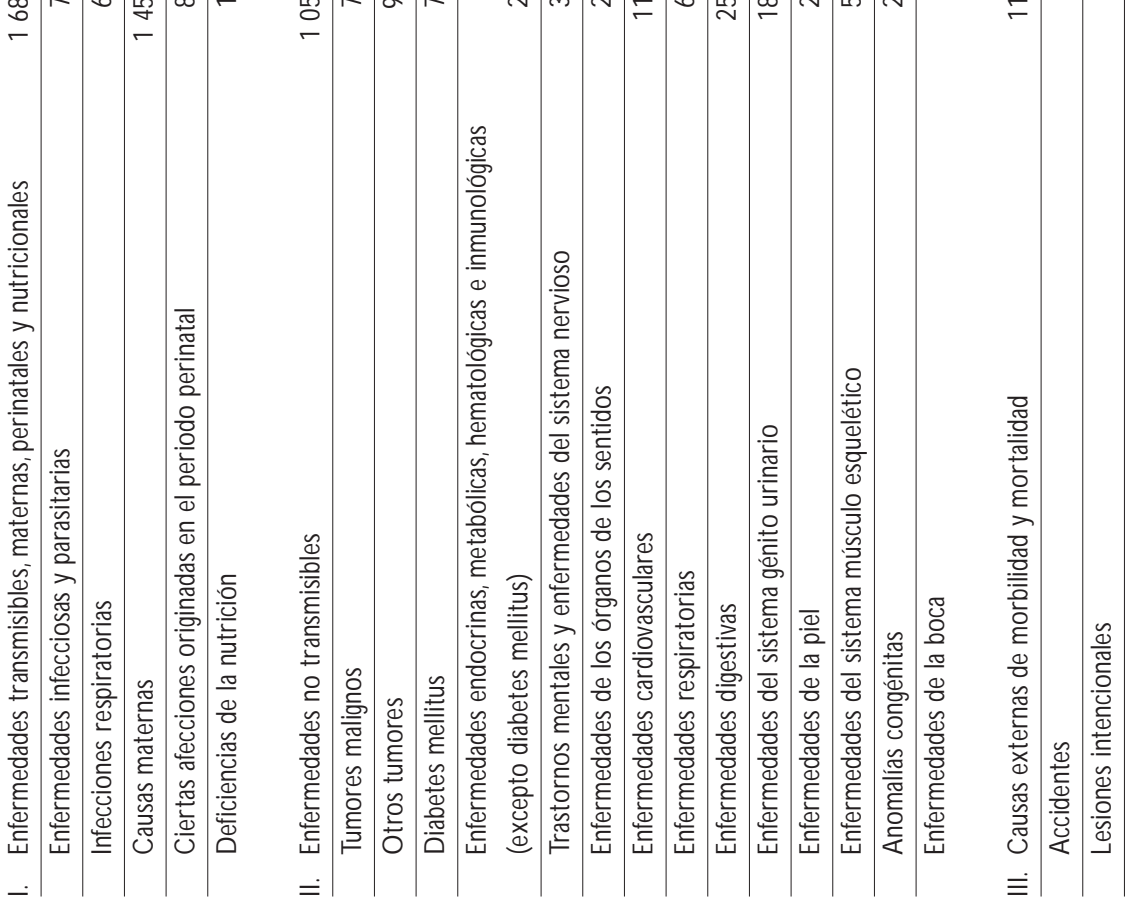


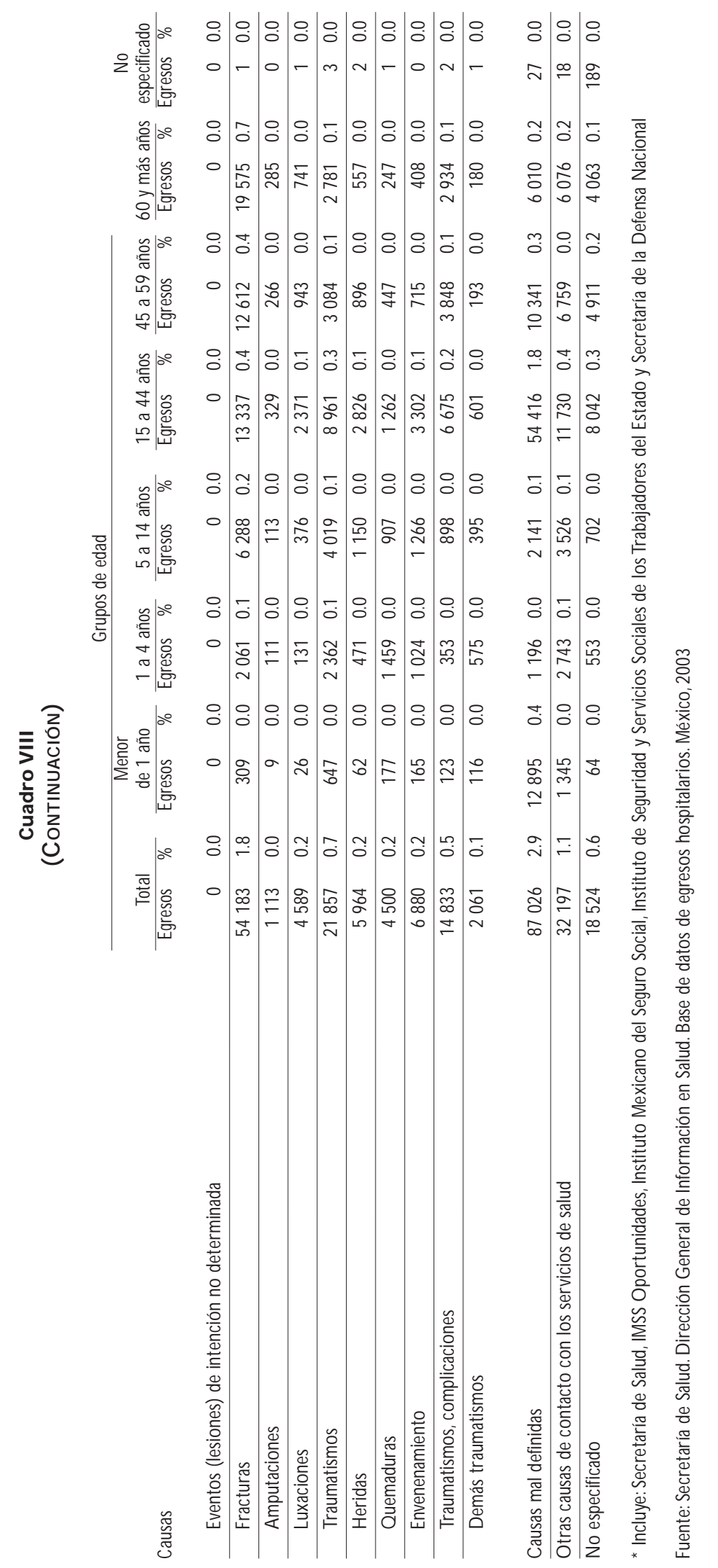




\section{Cuadro IX \\ Grandes grupos de causas de mortalidad hospitalaria, población derechohabiente.* Hombres. MÉXICO, 2003}

Causas

Egresos Tasa por $100000^{\ddagger} \quad \%$

Total

$37239 \quad 160$

100.0

I. Enfermedades transmisibles, maternas, perinatales y nutricionales

\begin{tabular}{rrr}
5944 & 26 & 16.0 \\
\hline 1826 & 8 & 4.9 \\
\hline 1616 & 7 & 4.3 \\
\hline 1840 & 8 & 4.9 \\
\hline 662 & 3 & 1.8
\end{tabular}

II. Enfermedades no transmisibles

\begin{tabular}{rrr}
28521 & 122 & 76.6 \\
\hline 4731 & 20 & 12.7 \\
\hline 330 & 1 & 0.9 \\
\hline 4739 & 20 & 12.7 \\
\hline 385 & 2 & 1.0 \\
\hline 677 & 3 & 1.8 \\
\hline 3 & 0 & 0.0 \\
\hline 7587 & 33 & 20.4 \\
\hline 2326 & 10 & 6.2 \\
\hline 4569 & 20 & 12.3 \\
\hline 1961 & 8 & 5.3 \\
\hline 105 & 0 & 0.3 \\
\hline 115 & & 0.3 \\
\hline 987 & 4 & 2.7 \\
\hline 6 & & 0.0
\end{tabular}

III. Causas externas de morbilidad y mortalidad

\begin{tabular}{rrr}
1432 & 6 & 3.8 \\
\hline 1006 & 4 & 2.7 \\
71 & 0 & 0.2 \\
19 & 0 & 0.1 \\
62 & 0 & 0.2 \\
\hline 2 & 0 & 0.0 \\
143 & 1 & 0.4 \\
10 & 0 & 0.0 \\
6 & 0 & 0.0 \\
\hline 31 & 0 & 0.1 \\
\hline 56 & 0 & 0.2 \\
\hline 26 & 0 & 0.1
\end{tabular}

Causas mal definidas

$\begin{array}{lll}439 & 2 & 1.2 \\ 903 & 4 & 2.4\end{array}$

No especificado

* Incluye: Instituto Mexicano del Seguro Social, Instituto de Seguridad y Servicios Sociales de los Trabajadores del Estado y Secretaría de la D efensa $\mathrm{N}$ acional

* Estimación realizada a partir de la muestra censal del Censo 2000 y las proyecciones de la población de México 2000-2030. Consejo Nacional de Población, 2003

Fuente: Secretaría de Salud. Dirección General de Información en Salud. Base de datos de egresos hospitalarios. México, 2003 


\section{Cuadro $X$ \\ Grandes grupos de causas de mortalidad hospitalaria, población derechohabiente.* Mujeres. México, 2003}

Causas Egresos Tasa por $100000^{\ddagger} \quad \%$

Total

$36566 \quad 153$

100.0

I. Enfermedades transmisibles, maternas, perinatales y nutricionales

$4901 \quad 20 \quad 13.4$
Enfermedades infecciosas y parasitarias Infecciones respiratorias Causas maternas

Ciertas afecciones originadas en el periodo perinatal

D eficiencias de la nutrición

\begin{tabular}{rrr}
1304 & 5 & 3.6 \\
1488 & 6 & 4.1 \\
\hline 85 & 0 & 0.2 \\
1467 & 6 & 4.0 \\
\hline 557 & 2 & 1.5
\end{tabular}

II. Enfermedades no transmisibles

\begin{tabular}{rrr}
29526 & 123 & 80.7 \\
\hline 5062 & 21 & 13.8 \\
\hline 368 & 2 & 1.0 \\
\hline 6205 & 26 & 17.0 \\
\hline 489 & 2 & 1.3 \\
\hline 512 & 2 & 1.4 \\
\hline 5 & 0 & 0.0 \\
\hline 8261 & 34 & 22.6 \\
\hline 1986 & 8 & 5.4 \\
\hline 3613 & 15 & 9.9 \\
\hline 1737 & 7 & 4.8 \\
\hline 126 & 1 & 0.3 \\
\hline 302 & 1 & 0.8 \\
\hline 852 & 4 & 2.3 \\
\hline 8 & & 0.0
\end{tabular}

Tumores malignos

III. Causas externas de morbilidad y mortalidad

\begin{tabular}{rrr}
788 & 3 & 2.2 \\
\hline 485 & 2 & 1.3 \\
22 & 0 & 0.1 \\
12 & 0 & 0.0 \\
89 & 0 & 0.2 \\
\hline 2 & 0 & 0.0 \\
71 & 0 & 0.2 \\
2 & 0 & 0.0 \\
2 & 0 & 0.0 \\
\hline 10 & 0 & 0.0 \\
\hline 77 & 0 & 0.2 \\
16 & 0 & 0.0
\end{tabular}

C ausas mal definidas

$\begin{array}{lll}407 & 2 & 1.1 \\ 944 & 4 & 2.6\end{array}$

No especificado

* Incluye: Instituto Mexicano del Seguro Social, Instituto de Seguridad y Servicios Sociales de los Trabajadores del Estado y Secretaría de la Defensa $\mathrm{N}$ acional

‡ Estimación realizada a partir de la muestra censal del Censo 2000 y las proyecciones de la población de México 2000-2030. Consejo Nacional de Población, 2003

Fuente: Secretaría de Salud. Dirección General de Información en Salud. Base de datos de egresos hospitalarios. México, 2003 


\section{Cuadro XI \\ Grandes grupos de causas de mortalidad hospitalaria, población no derechohabiente.* \\ Hombres. MÉxico, 2003}

Causas

Egresos Tasa por $100000^{\ddagger} \quad \%$

Total

$22436 \quad 100.0$

I. Enfermedades transmisibles, maternas, perinatales y nutricionales

\begin{tabular}{rrr}
7250 & 25 & 32.3 \\
\hline 1977 & 7 & 8.8 \\
\hline 982 & 3 & 4.4 \\
\hline 3243 & 11 & 14.5 \\
\hline 1048 & 4 & 4.7
\end{tabular}

II. Enfermedades no transmisibles

\begin{tabular}{rrr}
12422 & 44 & 55.4 \\
\hline 1225 & 4 & 5.5 \\
142 & & 0.6 \\
\hline 1504 & 5 & 6.7 \\
\hline 342 & 1 & 1.5 \\
713 & 2 & 3.2 \\
2754 & 10 & 12.3 \\
\hline 1023 & 4 & 4.6 \\
\hline 2951 & 10 & 13.2 \\
757 & 3 & 3.4 \\
\hline 25 & 0 & 0.1 \\
\hline 39 & 0 & 0.2 \\
\hline 933 & 3 & 4.2 \\
14 & 0 & 0.1
\end{tabular}

III. Causas externas de morbilidad y mortalidad

\begin{tabular}{ll} 
Accidentes & 1266 \\
Lesiones intencionales & 231 \\
\hline Eventos (lesiones) de intención no determinada & \\
\hline Traumatismos & 62 \\
Heridas & 1 \\
Envenenamiento &
\end{tabular}

Causas mal definidas

No especificado

\begin{tabular}{rrr}
587 & 6 & 7.1 \\
\hline 266 & 4 & 5.6 \\
\hline 231 & 1 & 1.0 \\
82 & 0 & 0.4 \\
6 & 0 & 0.0 \\
1 & 0 & 0.0 \\
1 & 0 & 0.0 \\
262 & 1 & 1.2 \\
915 & 3 & 4.1
\end{tabular}

* Incluye: sólo a la Secretaría de Salud, datos no disponibles para IMSS-0 portunidades

₹ Estimación realizada a partir de la muestra censal del Censo 2000 y las proyecciones de la población de México 2000-2030. Consejo Nacional de Población, 2003

Fuente: Secretaría de Salud. Dirección General de Información en Salud. Base de datos de egresos hospitalarios. México, 2003 


\section{Cuadro XII \\ Grandes grupos de causas de mortalidad hospitalaria, POblación no derechohabiente.* Mujeres. MéXICO, 2003}

Causas Egresos Tasa por $100000^{\ddagger} \quad \%$

Total

18014

63

100.0

I. Enfermedades transmisibles, maternas, perinatales y nutricionales

\begin{tabular}{rrr}
5530 & 19 & 30.7 \\
\hline 1254 & 4 & 7.0 \\
798 & 3 & 4.4 \\
\hline 229 & 1 & 1.3 \\
\hline 2476 & 9 & 13.7 \\
773 & 3 & 4.3
\end{tabular}

II. Enfermedades no transmisibles

$11160 \quad 39 \quad 62.0$

Tumores malignos

1233

0 tros tumores

1233

Diabetes mellitus

1932

Enfermedades endocrinas, metabólicas, hematológicas e inmunológicas (excepto diabetes mellitus)

Trastornos mentales y enfermedades del sistema nervioso

343

Enfermedades cardiovasculares

$\begin{array}{lll}321 & 1 & 1.8\end{array}$

Enfermedades respiratorias

$\begin{array}{lll}3193 & 11 & 17.7\end{array}$

Enfermedades digestivas

$\begin{array}{lll}950 & 3 & 5.3\end{array}$

Enfermedades del sistema génito urinario

1484

Enfermedades de la piel

Enfermedades del sistema músculo esquelético

A nomalías congénitas

Enfermedades de la boca

$\begin{array}{lll}602 & 2 & 3.3\end{array}$

$\begin{array}{lll}43 & 0 & 0.2\end{array}$

$102 \quad 0.6$

$\begin{array}{rrr}795 & 3 & 4.4 \\ 4 & 0 & 0.0\end{array}$

III. Causas externas de morbilidad y mortalidad

Accidentes

\begin{tabular}{rrr}
502 & 2 & 2.8 \\
\hline 416 & 1 & 2.3 \\
43 & 0 & 0.2 \\
33 & 0 & 0.2 \\
1 & 0 & 0.0 \\
7 & 0 & 0.0 \\
1 & 0 & 0.0 \\
1 & 0 & 0.0
\end{tabular}

Causas mal definidas

$\begin{array}{lll}184 & 1 & 1.0 \\ 638 & 2 & 3.5\end{array}$

No especificado

638

* Incluye: sólo a la Secretaría de Salud, datos no disponibles para IMSS-0 portunidades

₹ Estimación realizada a partir de la muestra censal del Censo 2000 y las proyecciones de la población de México 2000-2030. Consejo Nacional de Población, 2003

Fuente: Secretaría de Salud. Dirección General de Información en Salud. Base de datos de egresos hospitalarios. México, 2003 


\section{Cuadro X III \\ Mortalidad hospitalaria por GRANDES GRUPOS de CAUSA, SEgún INSTITUCión. Hombres. Sector público del sistema nacional de Salud,* MéXico, 2003}

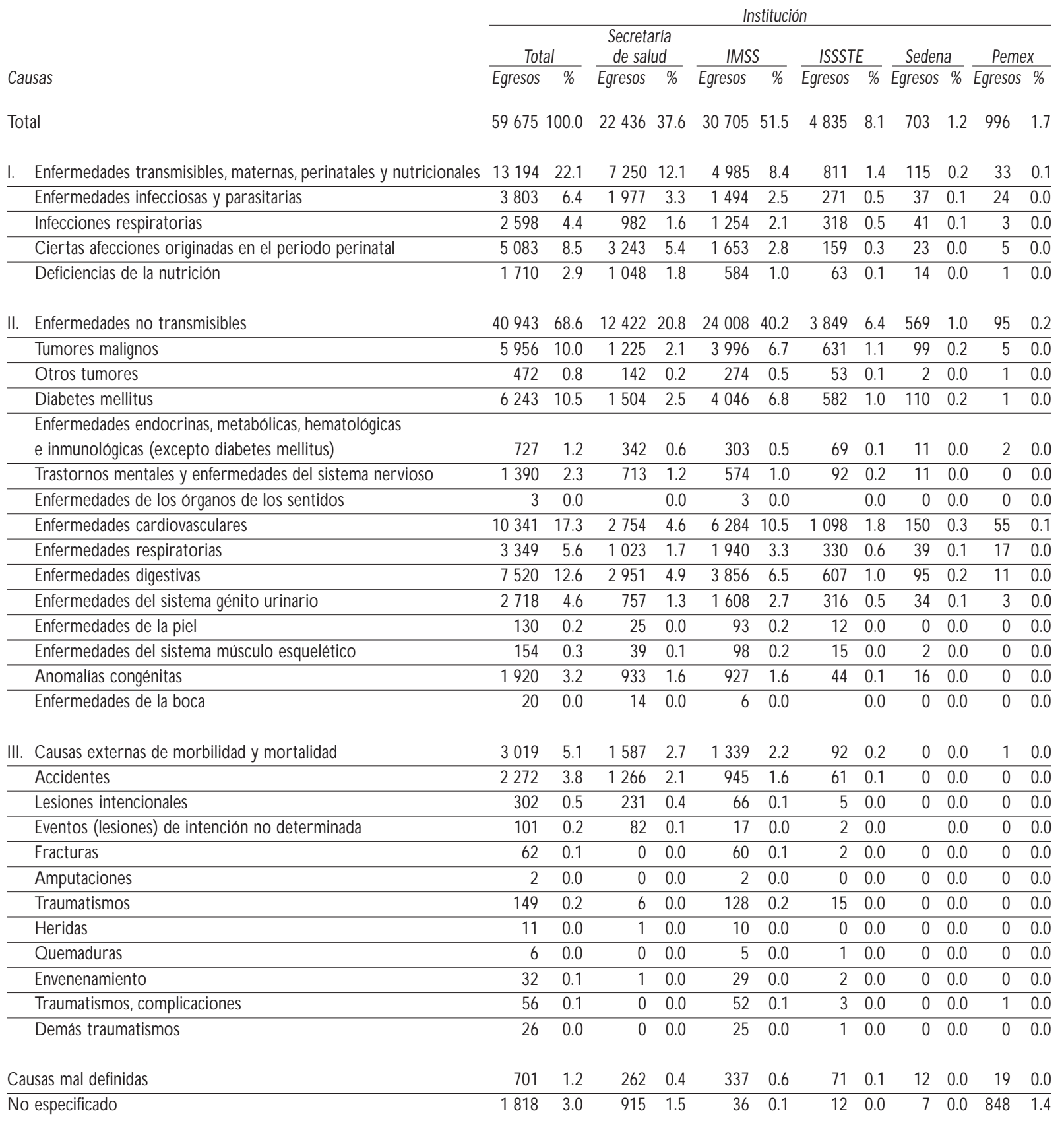

* Incluye: Secretaría de Salud, Instituto Mexicano del Seguro Social, Instituto de Seguridad y Servicios Sociales de los Trabajadores del Estado y Secretaría de la D efensa $\mathrm{N}$ acional

IMSS: Instituto Mexicano del Seguro Social

ISSSTE: Instituto de Seguridad y Servicios Sociales de los Trabajadores del Estado

Sedena: Secretaría de la D efensa N acional

Pemex: Petróleos Mexicanos

Fuente: Secretaría de Salud. Dirección General de Información en Salud. Base de datos de egresos hospitalarios. México, 2003 


\section{Cuadro XIV \\ Mortalidad hospitalara por Grandes Grupos de CAUSA, SEgÚn institución. Mujeres. Sector público del Sistema Nacional de Salud,* México, 2003}

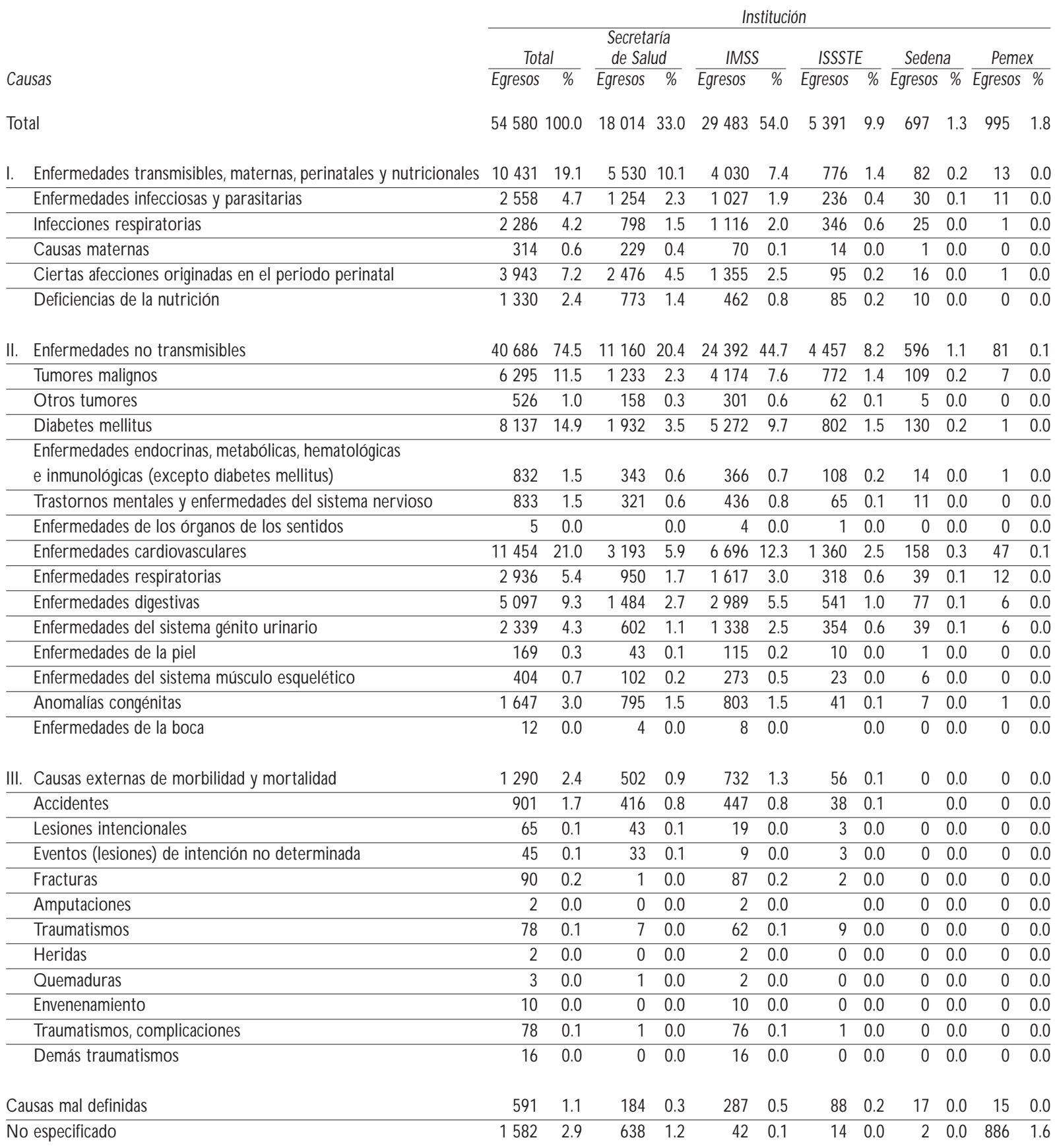

* Incluye: Secretaría de Salud, Instituto Mexicano del Seguro Social, Instituto de Seguridad y Servicios Sociales de los Trabajadores del Estado y Secretaría de la D efensa $\mathrm{N}$ acional

IMSS: Instituto Mexicano del Seguro Social

ISSSTE: Instituto de Seguridad y Servicios Sociales de los Trabajadores del Estado

Sedena: Secretaría de la D efensa N acional

Pemex: Petróleos Mexicanos

Fuente: Secretaría de Salud. Dirección General de Información en Salud. Base de datos de egresos hospitalarios. México, 2003 


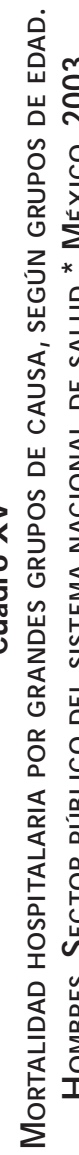

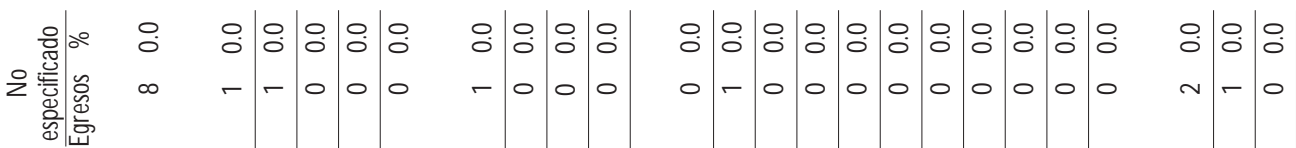

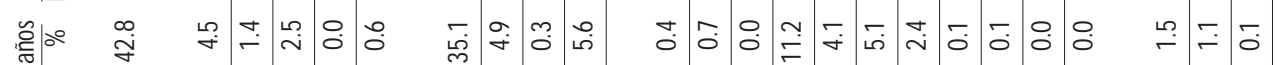

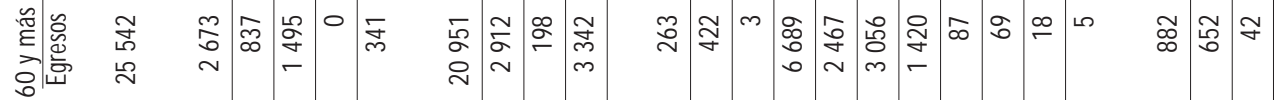

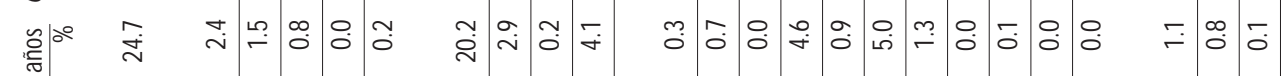

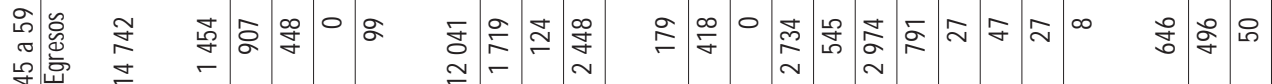

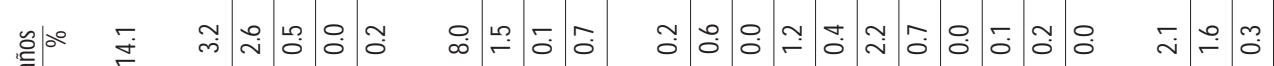

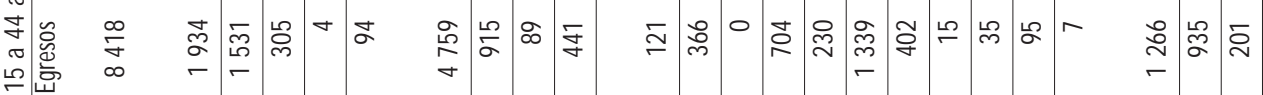

这

在

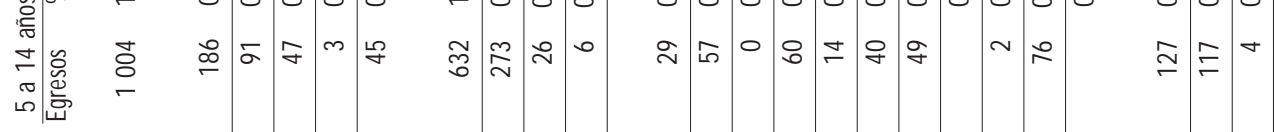

원 䢠

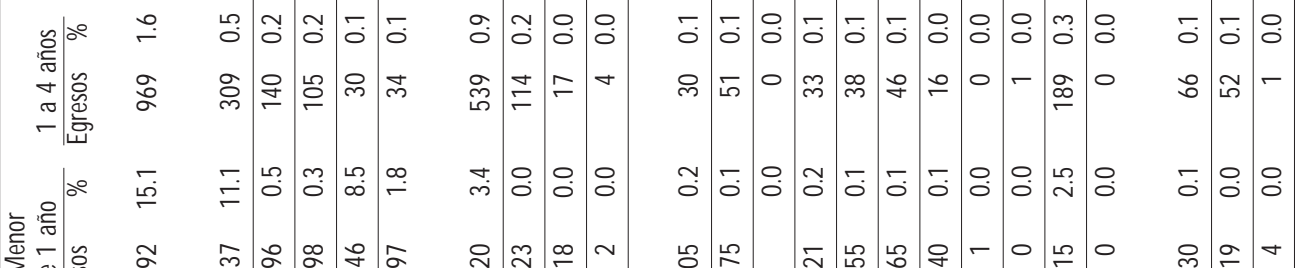

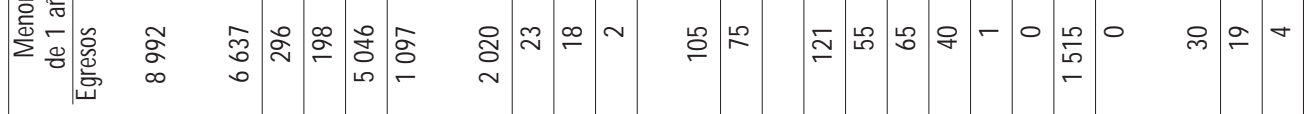

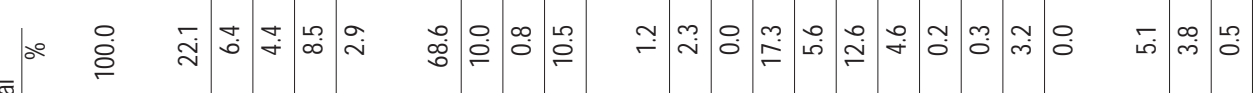

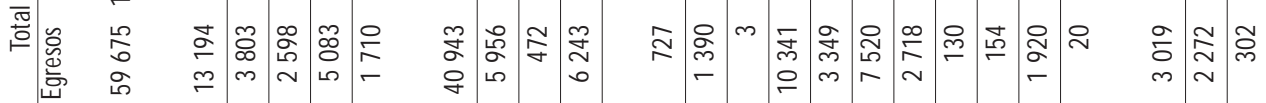
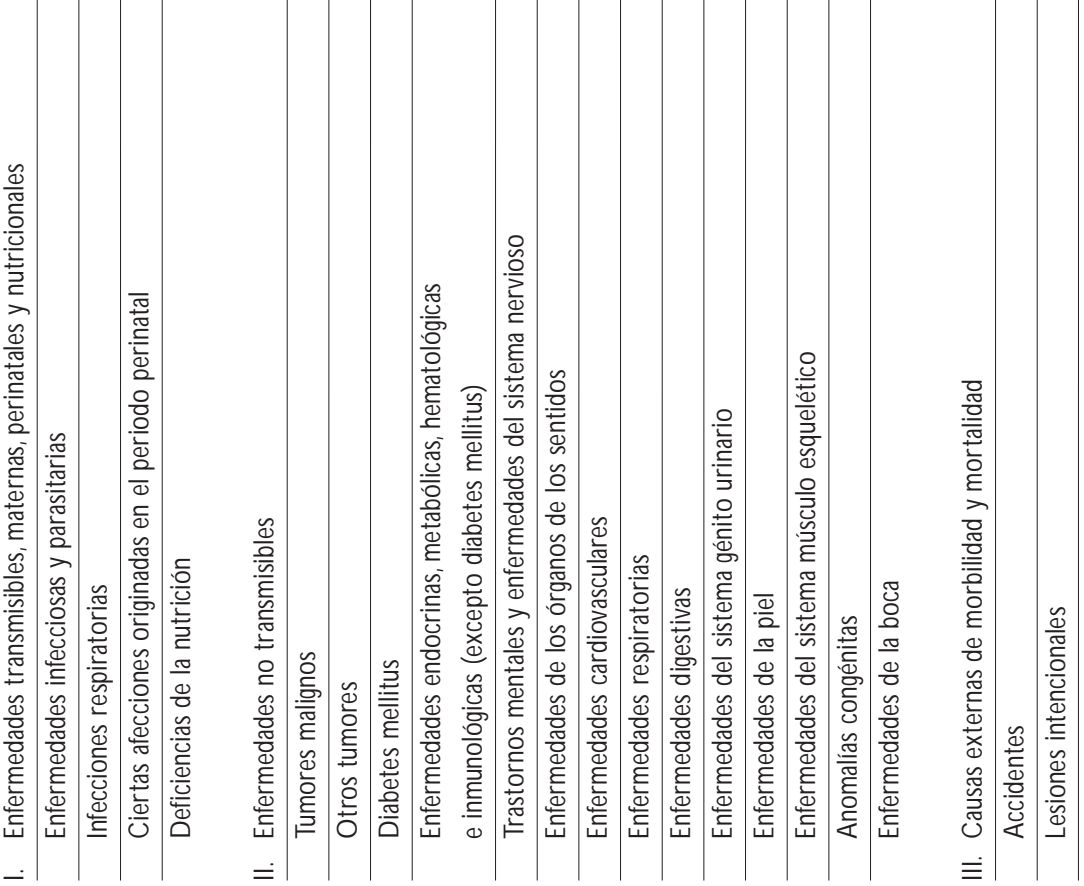


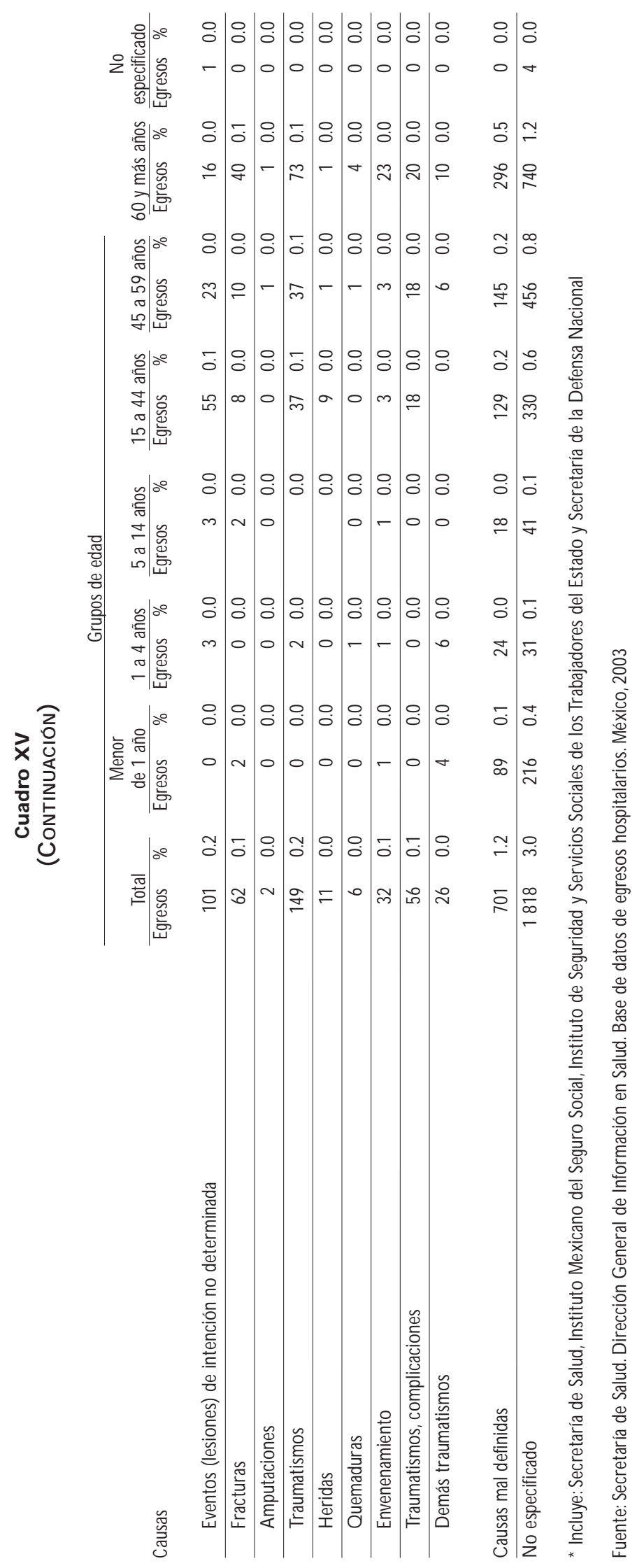




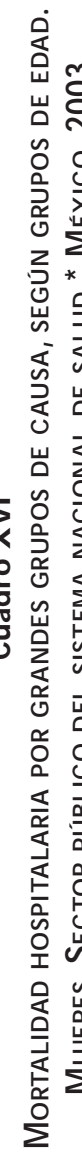

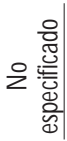

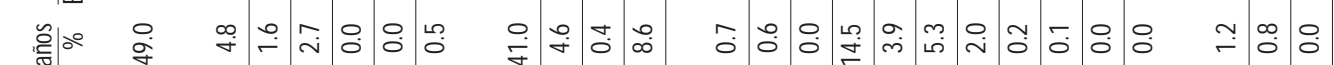

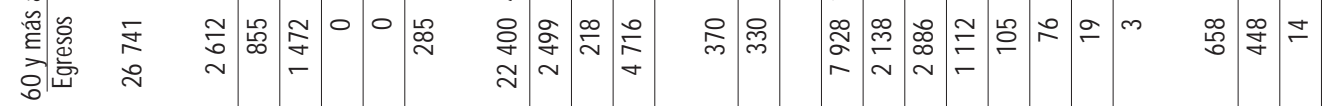

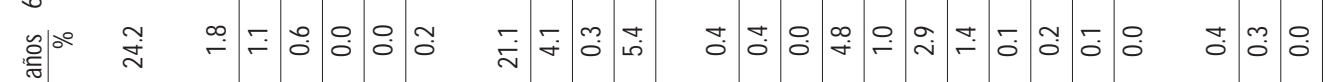

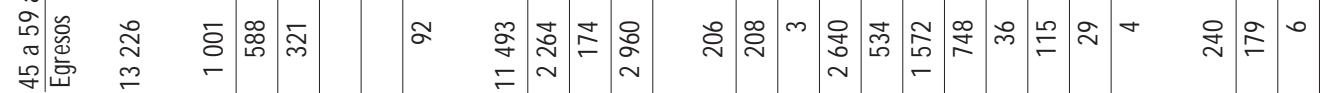

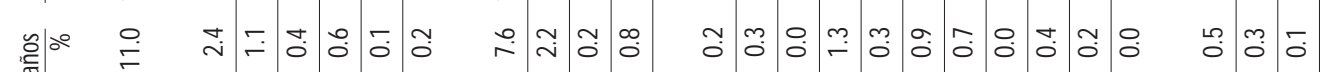

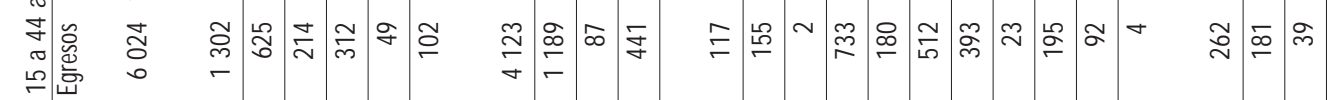

ก

g

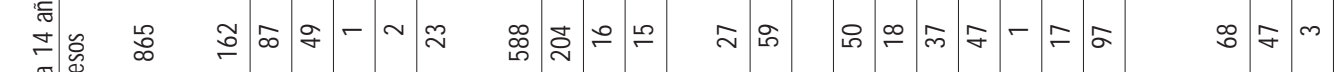

:

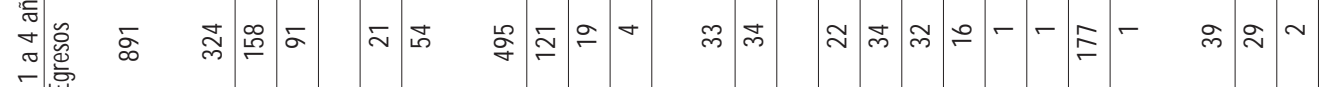

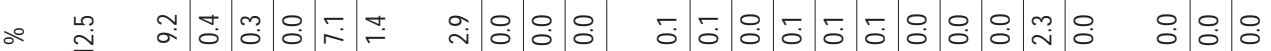

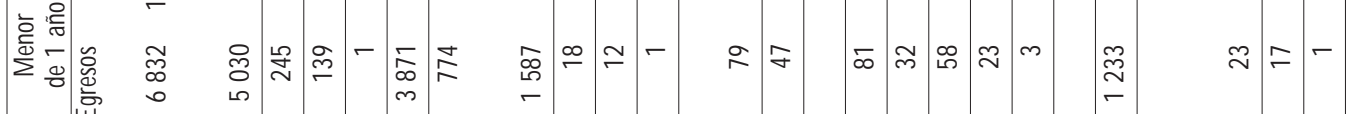

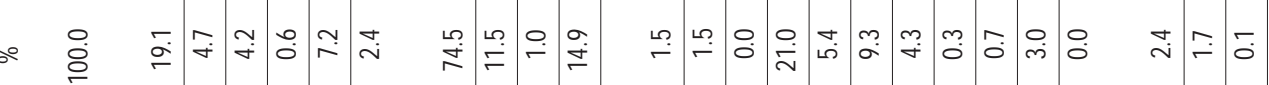

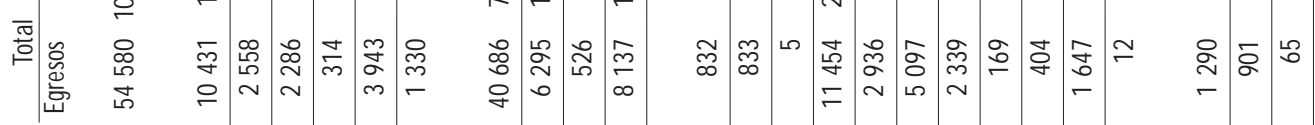
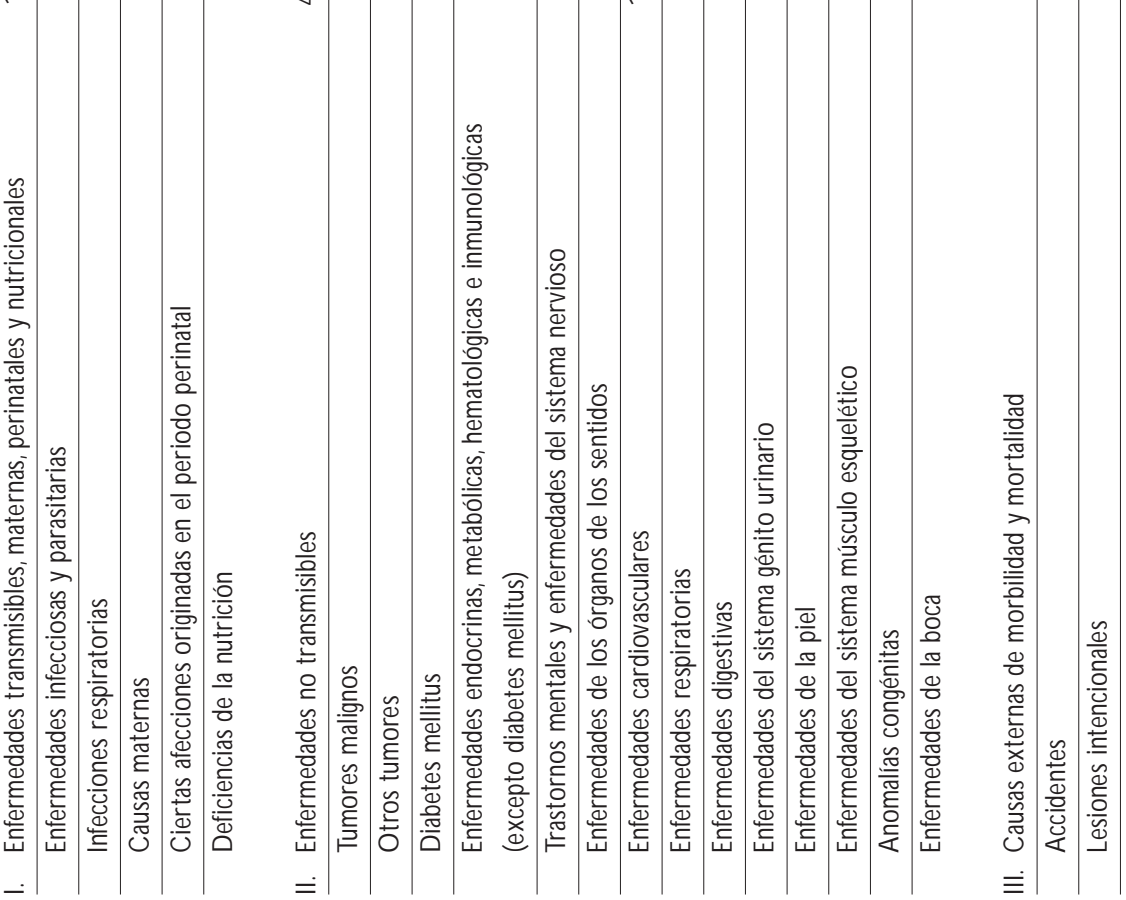


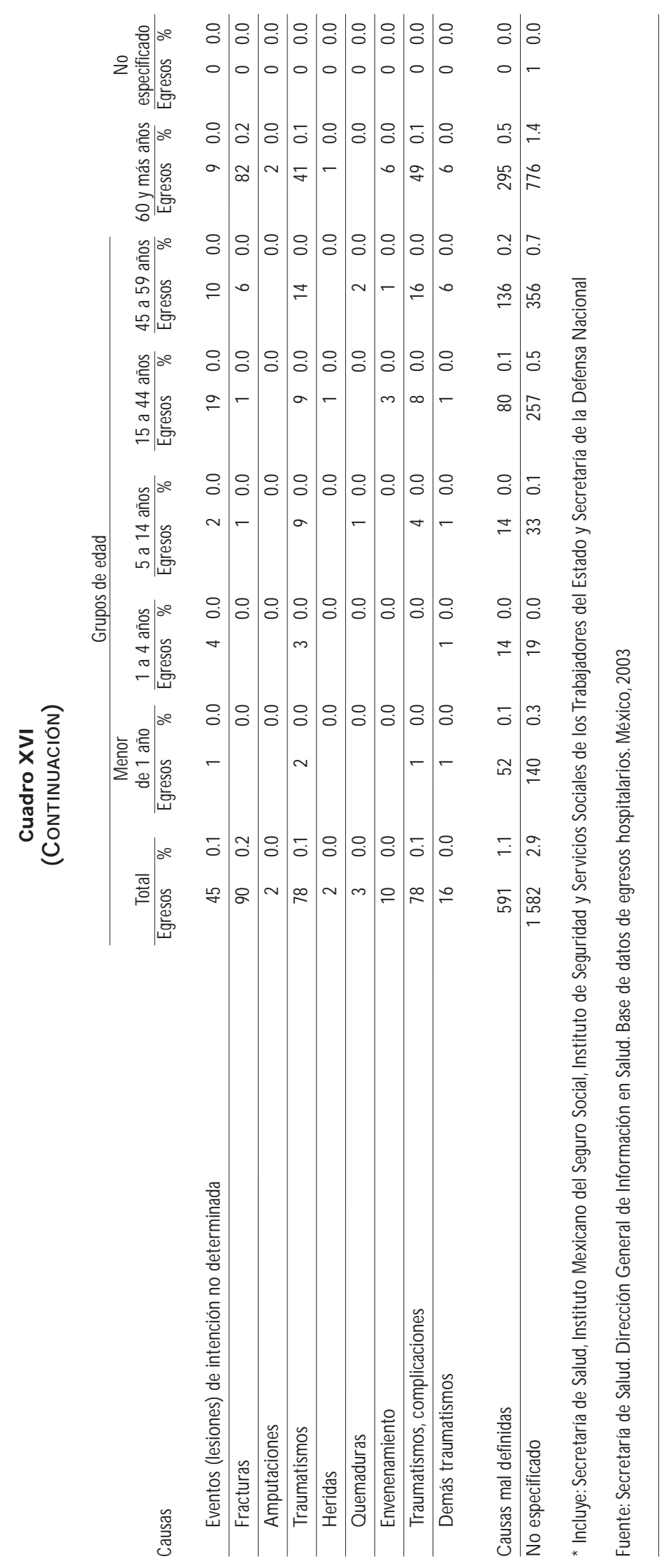

